\title{
EFTEM Contrast Tuning and EELS Fine Structure Analysis for Characterization of Carbon Containing Ultra-Low-k Dielectric Materials
}

\author{
Brenda Prenitzer ${ }^{1}$, Stephen Schwarz ${ }^{1}$, Brian Kempshall ${ }^{1}$, Imen Rezadad ${ }^{1,2}$
}

1. NanoSpective, Inc., Orlando, Florida, USA.

2. Physics Department, University of Central Florida, Orlando, Florida, USA.

Miniaturization of transistors is but a single leg of the race to improve speed in integrated circuits (IC). Interconnect is equally as crucial to the performance of advanced technology node devices. As production devices are emerging at the $14 \mathrm{~nm}$ node, there is a corresponding and necessary reduction in interconnect pitch. Blazing fast processing speeds and densely packed routing intensifies the imperative to use advanced ultra-low-k (ULK) dielectric materials to reduce the RC time delay, minimize crosstalk and lower power consumption. Technological evolution has ushered in the integration of progressively lower dielectric constant materials starting with fluorinated dense materials through carbon-laden polymers, organically modified $\mathrm{SiO}_{x}$ and into the era of porous silicate materials with incorporated organic species. While these porous insulators have proven to perform admirably, there are concurrent collateral complexities for process integration and as well as for characterization. These materials are less thermally and mechanically robust than their traditional $\mathrm{SiO}_{\mathrm{x}}$ predecessors. Nevertheless, the reliability of the IC is dependent on the ability of the dielectric to maintain its integrity through the severe processing steps and beyond. ${ }^{1}$ A multitude of characterization opportunities are generated by the use of ULK materials in the interlevel dielectric (ILD) and the required complex support network of films for diffusion barrier, adhesion, etch stop to name a few.

Some properties of interest, e.g., overall morphology, composition, etc. are handily conquered by conventional analytical microscopy; however, some of the most important properties of ultra-low-k dielectrics are dependent on the pore structure, permittivity, density and chemistry. Methods that are well suited for thin film analysis on a blanket wafer find their limits on a fully processed wafer or die. In general, most characterization methods either lack sufficient sensitivity or lateral spatial resolution to adequately characterize ULK dielectrics. Ever increasing sophistication in tools and innovation in techniques is required to keep pace. Electron energy loss spectroscopy (EELS) and energy filtered transmission electron microscopy (EFTEM) are applied to the characterization of the ILD and etch stop layers (ESL) of ICs at a technology node of $32 \mathrm{~nm}$ or smaller. EELS is used to probe valence and conduction bands to determine critical information about the local bonding environment of Si, O, N, C and $\mathrm{H}$ in ULK and ESL layers. The spectra can be used as a chemical fingerprint. Examples of contrast enhancement using EFTEM contrast tuning in carbon containing materials will be shown. Direct imaging of nanometer size pores through the thickness of the TEM specimen is confounded by the fact that the image is a $2 \mathrm{D}$ projection of a $3 \mathrm{D}$ volume. With pore sizes on the order of $1-2 \mathrm{~nm}$, the projected volume will contain the superposition of several pores. Filtered imaging enhances the ability to observe the pore structure in ULK dielectrics by forming images with the relatively few electrons that have suffered inelastic interactions corresponding to the selected energy of position of the spectrometer slit.

\section{References}

[1] Sheng-Wen Chen et al., Materials 5 (2012), 377-384

[2] James M. Howe, et al., Journal of Electron Microscopy 53(4) (2004), 339-351 


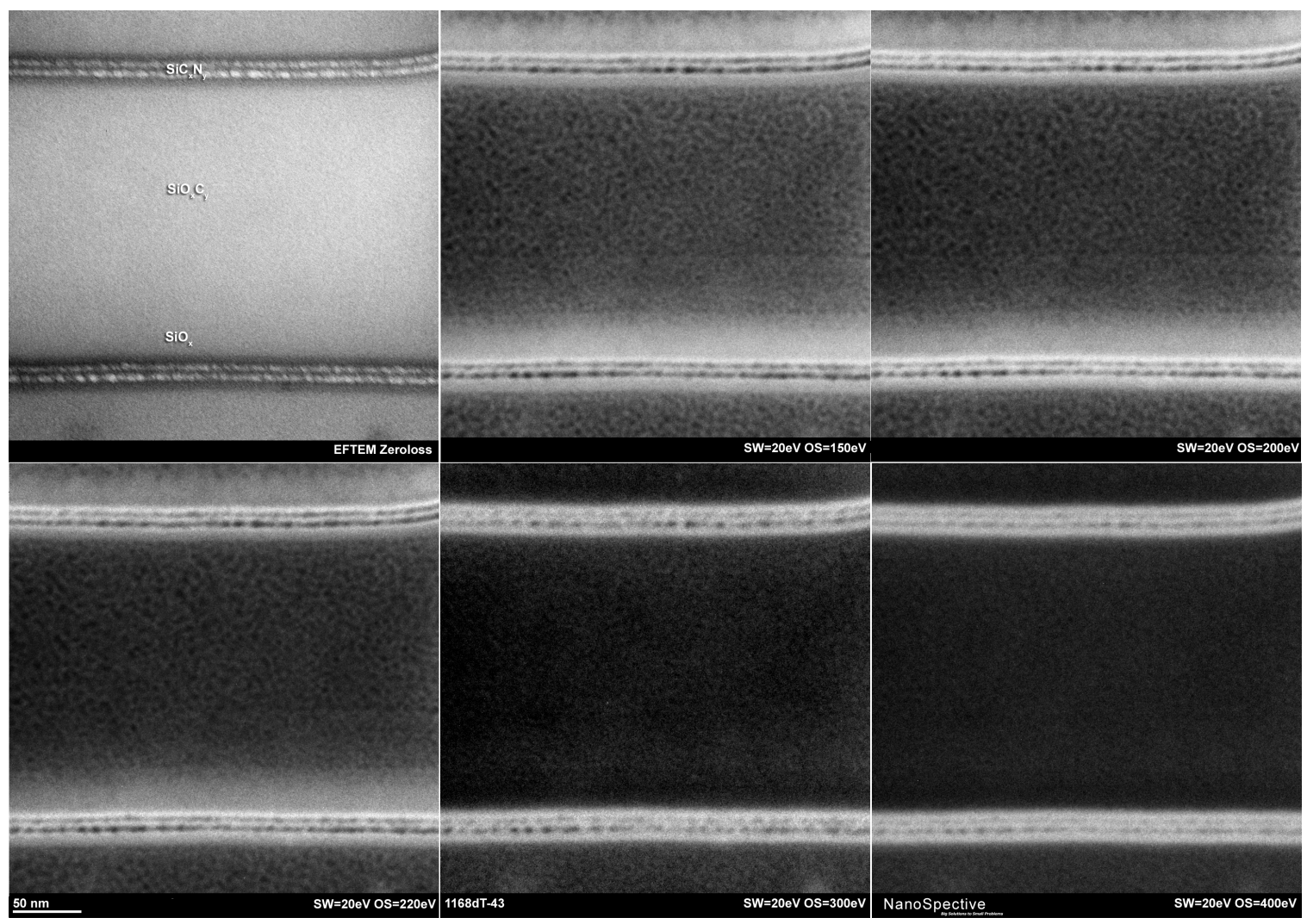

Figure 1 EFTEM energy filtered series. The enhanced contrast allows the direct observation of the multilayered dielectric structures as well as the porosity in the ILD.

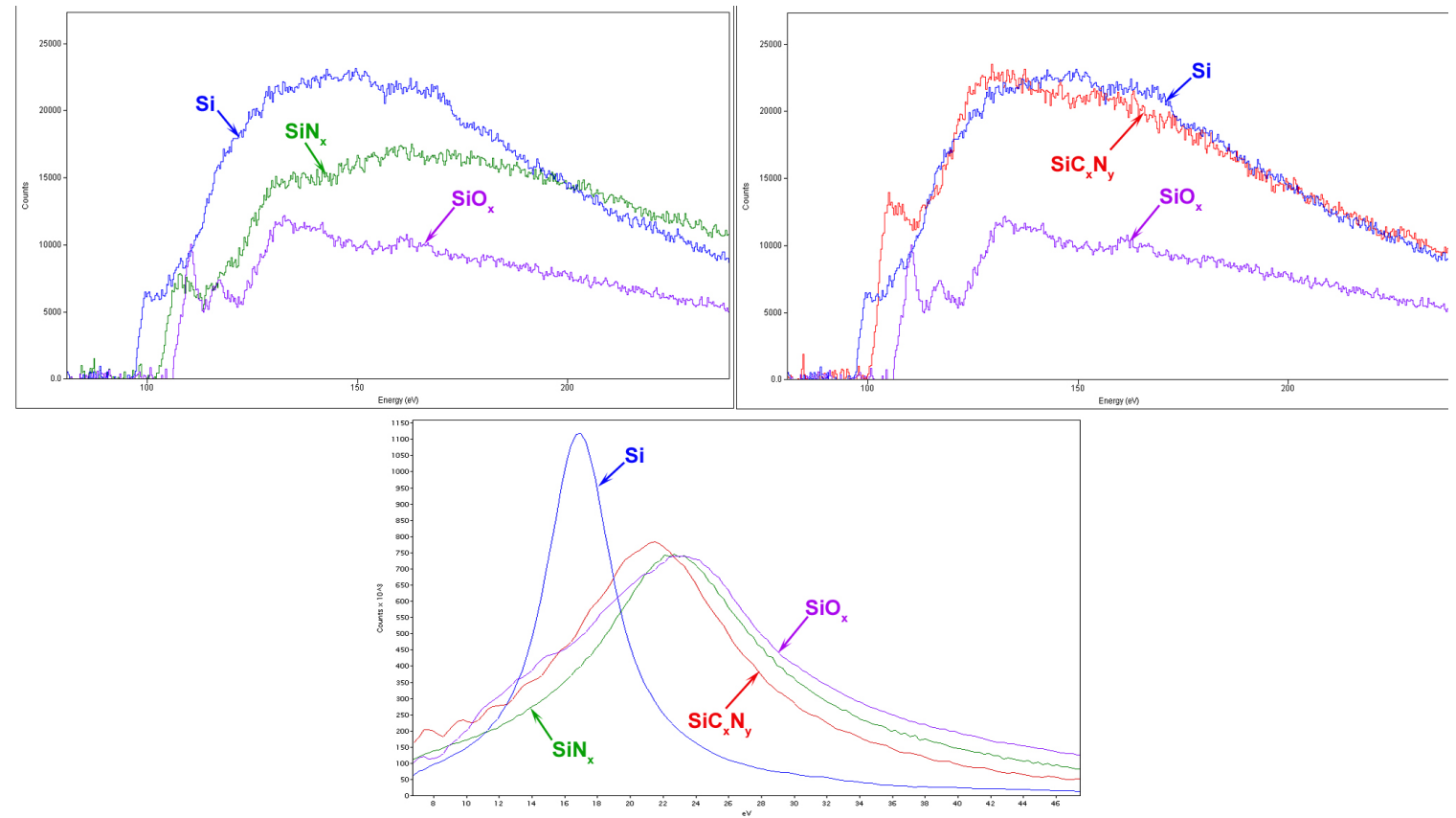

Figure 2. Low and core electron energy loss spectra. The fine structure for each material is distinctive and is indicative of how the density of unoccupied states changes with atomic bonding. 